\title{
Política macroeconómica y concertación
}

\author{
Carolina A. de Franco
}

\section{Introduccion}

Con la firma de los Acuerdos de Paz en Chapultepec el 16 de enero de este año, y con el cese al enlrentamiento armado a partir del primero de lebrero recién pasado, se logra poner fin a una larga guerra que generó violencia, destrucción y muerte en el pals, y que marcó a $\mathrm{EI}$ Salvador con una de las partes más sangrientas de su historia.

Pero ahora que la alegria general se va apaciguando, queda la ardua tarea de reconslruir el pais, y de llevarlo por la senda de un desarrollo económico que muchos han expresado debe ser: "fuerte, sostenido y con equidad".

Existe bastante acuerdo sobre la lorma de desarrollo que se busca, pero no es Iácil alcanzarlo en la realidad. Hay mucho mayor consenso en relación al objetivo úllimo al que habrán de orienlarse lodos los esfuerzos en pos de una sociedad mejor - sobre todo en lo que se refiere a los dos primeros atributos del desarrollo: "fuerte y sostenido"- que al respecto de los insirumenlos, políticos, y pasos a emprender para lograrlo. Divergencias que muchas veces, aunque no exclusivamente, se generarán de atribuir dislintos énlasis e importancia a los componentes que debe presenlar una sociedad que se vanaglorie de ser económicamente fuerte y que presente un alto grado de juslicia social. Por tanto, los caminos propueslos para llegar a esle lipo de sociedad pueden ser extremadamente distintos. 
La exislencia de diterentes ideologlas, los antagonismos, resentimienlos y diferentes intereses, obviamente hacen aún más complicado alcanzar un consenso en relación a la manera en que debe manejarse el destino del pals. Pero es precisamente para llegar a un acuerdo mínimo en materia económica y social entre los seclores empresarial, gubemamental y laboral que se crea el Foro de Concertación Económico y Social'. Este organismo tendrá ante sí la espinosa faena de lograr un consenso entre grupos cuyos intereses han sido tradicionalmente antagónicos.

Por la generalidad con que se tratan algunos problemas o sus soluciones en los Acuerdos o por la omisión de olros asunlos que demandan urgentemente de un resultado concertado ${ }^{2}$, las expectalivas descansan ahora en el Foro de Concertación Económico y Social, al que se mira como el mecanismo a través del cual podrá llegarse - luego de una serie de concesiones por los distintos agentes económicos- a un acuerdo en torno a los principales problemas económicos y sociales existentes y a la forma de como resolverlos.

El propósito de este arliculo es presentar algunas reflexiones en torno a los límites de concertación en materia de gestión macroecónomica, y dar algunas propuestas para una gestión macroeconómica de consenso que puede surgir del Foro.

II. Concepto de Gestlón Macroeconómica e Importancla de lograr un consenso en torno a ella.

Podemos definir en forma general la gestión macroeconómica como el conjunto de medidas, pasos e instrumentos -que idealmente deben conformar todo un cuerpo sistemático en torno a un objeto y eslrategia de desarrollo preciso- del que el gobiemo hace uso para influir en los

1. La creación de este organismo está contemplada en el numeral ocho del capltulo ocho de los Acuerdos de Nueva York, donde se estipula que tendrán participación igualitaria en el Foro los sectores gubernamentales, laboral y empresarial con el "objeto de lograr un conjunto de amplios acuerdos lendientes al desarrollo económico y social del pals, en beneticios de todos sus habitantes" ..."En términos generales, el Foro será el mecanismo para concertar medidas que alivien el cosio social del programa de ajusle estructural".

2. El capltulo $V$ de los Acuerdos de Nueva York eslá dedicado al tema económico y social, pero el énlasis en cuanto a lo económico se ha centrado en el problema agrario y en la tenencia de las tierras en las zonas en conflicto. aunque también incluye lineamientos para la asistencia crediticia y algunas medidas para aliviar el costo social de los programas de ajusle estructural. 
grandes agregados macroeconómicos es, a saber, el productor interno bruto, los elementos de la demanda, el nivel de precios, etc., con el objelo de mejorar la eliciencia de la economía y las condiciones sociales. y/o para hacer írenle a serios desequilibrios económicos.

La importancia de la gestión macroeconómica es obvia por cuanto a través de ella se puede influir en el desempeno económico, en el desarollo y crecimiento económico, y lo que es más importante aún, se puede contribuir a disminuir o a mejorar, según sea el caso, los índices de desigualdad económica y social.

La experiencia demuestra que no puede separarse fácilmente la polftica económica de la política social, la forma en que se utilicen los instrumenlos de política económica tendrá un impacto decisivo en las condiciones de la población. Más aún, el logro de un desempeno macroeconómico favorable no garantiza, que la mayorla goce de los benelicios de esle mejor desenvolvimiento global de la economía.

En el caso de las políticas de estabilización, las medidas implementadas para solventar los desequilibrios internos (inflación y déficit fiscal) y externos (déficit de la balanza de pagos) producen efectos contradictivos en la economia con graves costos sobre todo para las mayorias más pobres ${ }^{3}$.

Igualmenle, la restricción de recursos hace que la asignación de fondos y esiuerzos prioritariamente a algunas áreas, muchas veces se efectue a costa del detrimento de algunos sectores que pueden ser aquellos con mayor potencial para elevar el nivel de vida de los más necesilados. Así, por ejemplo, en el lado financiero, una parte imporlante de los escasos recursos se dirigen a las grandes empresas, y soblo una porción marginal logra ser absorbida por los pequenos negocios o actividades informales.

Por último, debe considerarse que en un país con una estructura distribuliva baslanle desigual se hace sumamente difícil lograr medidas reivindicalivas para la mayoria desposeida, quien ante su incapacidad de aspirar por la via legal a un mejor nivel de vida recurre muchas veces a la violencia o a la delincuencia, generándose un alto costo social. Por

3. En primer lugar, el cierre de empresas genera mayor desempleo, subempleo y actividades inlormales, menores salarios reales, y una concentración de la riqueza aún mayor; la disminución del gasto social público equivale a un deterioro de los ya precarios servicios de salud, educación y vivienda; la liberalización de precios y la eliminación de subsidios a los productos básicos disminuye los salarios reales y pone en situación más apremiante a los pobres. 
consiguiente, es de extrema importancia que dentro de la política económica se incorporen medidas especificamente disenadas para alterar el actual esquema de concentración y distribución de la riqueza y el ingreso, siempre y cuando esto se haga dentro de un marco legal, participativo y democrático.

\section{Limites de la concertaclón}

Obviamente si concertar requiere de consesiones de parte de agentes con distintos intereses, éslo impone ciertos límites a los acuerdos a que se puede llegar.

a. El programa de ajuste estructural y la necesidad de una estrategia de desarrollo económico-social clara

En el momento de presentar propuestas de concertación en materia económica en el Foro hay que recordar que, en base a los Acuerdos, exisle la aceptación de que se continuará con un sistema capitalisla de producción, y que el gobierno seguirá la implementación de su Programa de Ajuste Estructural. En esle sentido, es indispensable que antes de negociar se lenga un conocimiento básico de lo que el ajusle estruclural implica, lanto en su fase de estabilización como de cambio estructural4.

Consecuentemente, no se podrá cambiar de forma global el Programa del Gobierno, ni los lineamienlos generales del mismo: aperlura comercial, liberalización de precios, privalización, reducción del délicí fiscal, control de las variables monelarias-crediticias, y promoción de exportaciones - actividad a la que se le ha asignado un papel preponderante como mecanismo dinamizante de la economía. Pero eslo no signitica que no sea posible negociar la gradualidad, los malices y variantes que pueden tener cada una de estas medidas. De hecho el mismo gobiemo ha modificado bastante la implementación del programa que presentó al inicio de su gestión'.

4. Un buen análisis crítico del programa de Ajuste Estructural impulsado por el Fondo puede verse en Abrego Lisandro, "Los Programas de Ajuste del FMI: Contenido, bases térico-analíticas y resultados", Realidad Económico-Social, N²3, sep.-octu. 1991.

5. Los organismos internacionales que han olorgado londos condicionados a la implementación del Programa de Ajuste Estructural en los palses receptores, también han introducido algunas modificaciones a las formas de implementación del programa, con el objeto de corregir lallas o de distribuir más equitativamente los coslos del mismo. 
En relación a lo anterior deben identificarse las principales politicas macroeconómicas y analizar en lorno a cada una de ellas su impacto en el crecimiento y el desarrollo económico, en las condiciones de vida de los más pobres, y en los esquemas de concentración y distribución del ingreso y la riqueza. Esto es, bajo el supuesto que el combale a la pobreza ha de eslar presente en cada una de las medidas emprendidas por el gobierno, tema del cual se hablará más adelante.

La tarea anterior será realizada más eficazmente si se cuenta con una visión global acerca de la nueva eslralegia generai de desarrollo a seguir, y de las condiciones fundamentales que son indispensables para que cualquier eslralegia de desarrollo funcione: un minimo de estabilidad económica, un abastecimiento seguro y suficiente de divisas, incremento de la productividad, elc.

Pero es precisamente la falla de claridad sobre la actividad que se convertirá en el componente impulsador del crecimiento lo que hace aún más ditícil el logro de una gestión macroeconómica verdaderamente humana. Ante un consenso general desde hace varios años sobre el deterioro del modelo agroexporlador y de su papel impulsador del desarrollo económico y social, nos encontramos con que tanto dentro del modelo neoliberal como en los modelos allernativos, hay mucho menos claridad y bastante divergencia en cuanto a la actividad que se convertirá en el componente impulsador del crecimiento, y sobre la forma en que debe encauzarse.

Esto indudablemente no permite lograr el cuerpo de políticas económicas más idóneo para reactivar la economía en poco tiempo y en forma firme y sostenida ${ }^{6}$; pero ante las limitaciones del marco denlro del cual se ha de negociar, deben identificarse aquellas políticas macroeconómicas cuyos efectos directos o colaterales tienen un alto costo para la población más pobre del pais, y que generan mayor concentración y desigual distribución del ingreso. Simultáneamente, deben analizarse las modificaciones a politicas y las implementación de políticas alternativas que se propondrán aprovechando el espacio favorable para negociar que se presenta en esla ocasión, donde no podrán rechazarse propuestas -como se hizo en el pasado- aduciendo el conflicto armado como el principal limitanle para emprender algunas medidas de gestión económica con carácler social.

6. El lector interesado en ahondar más sobre los pilares de crecimiento sobre los que han descansado los modelos económicos de los países centroamericanos puede relerirse a Bulmer-Thomas, Sludies in the Economics of Central America, Londres, Macmillan Press, 1988. 


\section{b. La Cooperación Internacional}

La enorme alluencia de fondos promedios por organismos internacionales y gobiernos extranjeros para ayudar a la reconstrucción y recuperación del pais, facilitan la tarea de lograr un crecimiento económico con equidad, pero al mismo tiempo imponen algunos condicionamienlos para todas las partes.

Para tener una mejor idea de esla clase de cooperación a continuación se citan los lineamientos que, de acuerdo al Minislerio de Cooperación Económica de Alemania, han de regir la cooperación alemanas para el desarrollo?.

- Concentración de la ayuda en la lucha contra la pobreza y la prolección ambiental como marco referencial permanente de la política alemana de desarrollo, segpun el cual deben orientarse el resto de los puntos principales y áreas de trabajo, en la medida en que sea posible y factible.

- Orientación del lipo y el volumen de la ayuda a un pais según las condiciones básicas para la realización de una estrategia de desarrollo orientada conlra la pobreza.

Los paises receplores de ayuda tendrán que cumplir requisitos mínimos en torno $a^{8}$ :

- El respelo a los derechos humanos.

- Participación de la población en la vida política (libertad de asociación, prensa, etc.).

- Seguridad Juridica

- Creación de un orden de mercado (eslablecimiento de precios de mercado, compelencia, defensa de la propiedad privada).

- Orientación hacia el desarrollo de la actividad estatal.

Por lanto, el desembolso de fondos está sujelo a la implementación de un programa de ajuste estructural y con ello a la apertura a las relaciones internacionales, a la economia de mercado, a la privatización, un menor estado, elc., pero lambién está sujeto a emprender los pasos en la dirección correcla para alcanzar una sociedad pluralista y democrálica, donde los derechos humanos sean respelados plenamente.

7. Van de Sand, Klemens y Ralph M. Mohs, "Mayor credibilidad para la política de desarrollo", Desarrollo y Cooperación, N² 1/1992.

8. Ibid. 
En esle sentido, se presentan limiles para los distinlos agentes económicos, se favorece una economía de mercado con promoción del sector empresarial, pero al mismo tiempo se quiere hacer claro que han de escucharse con efectividad las demandas de las mayorias populares.

\section{Tipo de Desarrollo Económico-Soclal que Hay que Concertar}

Dentro de los límites expueslos anteriormenle, queda aún mucho espacio para discutir y concertar. El primer paso es detinir el tipo de desarrollo que se quiere implementar. $A$ un nivel de generalidad bastante amplio, este punto no representará mayor problema pues en los últimos liempos - ya sea por convicción, presión internacional, presión social, presión política o por proteslas populares - todos parecen querer llegar a un mismo tipo de desarrollo: "No se puede sefhalar que en un país existe verdadero desarrollo socio-economico, si un porcentaje importanle de la población conlinúa estando en una situación de miseria y pobreza"日. "Romper el circulo vicioso de la pobreza debe ser, por consiguiente, la tarea priorilaria del Estado en el sentido de crear los mecanismos necesarios y generar las oportunidades que le permilan a los grupos en extrema pobreza participar y dis/rular efectivamenle de lodos los beneficios del desarrollo económico y social"10.

Anle tal declaración, difícilmente puede imaginarse que las grandes mayorías desposeidas estén en contra de ello. Pero cuando en la práctica se implementan medidas económicas contrarias a los intereses de los sectores populares, o cuando se dejan de emprender medidas disenadas específicamente para sacar a estos grupos de su condición de pobreza, surgen conflictos sociales y anlagonismos, cuya solución incluye la implementación de una gestión macroeconómica de consenso que busque un lipo de desarollo también "consensuado".

En términos generales podemos hablar de tres lipos de desarrollo":

1) El primero es aquel que afirma que los frulos del crecimiento llegarán a los pobres después de cierto tiempo, en forma automática, a Iravés de la generación de empleos. Aquí, son las mismas fuerzas del mer-

9. FUSADES, Informe Trimestral de Coyuntura № 2, Año 1991, p. 147.

10. Ibid.

11. Carrascolicea, Rosalba, Enrique Provencio y Carlos Tello, "La cuestión del Desarrollo en América Latina. Problemas, Referencias y Lineamientos", In vestigación Económica, Revista de la Facultad de Economía de la UNAM. № 194, Octubre-noviembre 1990. 
cado las que a la larga corregirán los problemas de pobrezas y desigualdad. Esle tipo de desarrollo también es conocido como la leorla del rebalse ${ }^{12}$.

2) En el segundo caso, conscientes de la insuliciencia del crecimiento económico para lograr equidad, se propone que a la par de la gestion macroeconómica para mejorar el desempeno, se pongan en marcha programas especialmente disenados para mejorar las condiciones de vida de los más pobres. El problema surge cuando las politicas macroeconómicas que se impulsan, producen efectos contrarios al bieneslar social.

3) Por úllimo tenemos al modelo de desarrollo que busca en forma primordial maximizar la distribución del ingreso y la riqueza, y superar la condición de pobreza de la población.

En el ambienle de democracia y justicia social que hoy prevalece, casi lodos sostienen el tercer lipo de desarrollo como modelo a implemenlar, aunque las acciones emprendidas pueden no estar en concordancia con lo que se afirma. En el caso del actual gobierno al programa que en la práctica ha implementado puede siluarse en la segunda forma de desarrollo, pero un consenso entre los distintos agentes sociales y económicos en cuanto a la gestión macroeconómica, puede llevarnos a implementar una clase de sociedad donde el hombre sea verdaderamente el centro del modelo, y se gire alrededor del combate a la pobreza.

En un pais donde la pobreza abarca a casi un $60 \%$ de la población, pobreza que tiene además un carácter crónico donde la mayoría de los pobres actuales son pobres por generación, con un empeoramiento de la siluación de miseria, y un aumento del número de pobres en los últimos afros $^{13}$, el ataque frontal a la pobreza ya no sólo se presenla como un deber moral, sino como condición indispensable para que pueda haber un desarrollo fuerte y soslenido.

Esto es muy importante de tener en cuenta en el momento en que se

12. Luego de que la hisloria se ha encargado repelida y contundentemente de demostrar la nula sustentabilidad del primer modelo de desarrollo como forma de aliviar, ya no digamos acabar, la pobreza, muy pocos son los que aún defienden esta tesis.

13. Para una estimación cuantitativa de la pobreza y de su carácler estructural ver Briones, Carlos "La Pobreza Urbana en El Salvador: Caracterlsticas y Diferencias de los Hogares Pobres 1980-1990". En este Irabajo se ensaya una nueva metodologla para medir la pobreza y el carácter permanente de la misma en El Salvador. 
analicen determinadas políticas -como la politica tributaria, política salarial, etc.- sobre las cuales el sector empresarial y el sector laboral pueden demandar medidas tolalmente opuestas, y donde la ganancia de uno de ellos se hace a costa de los menores benelicios, o mayores sacriticios del otro sector. Los agentes involucrados deben lograr mirar el contexto general de la situación en que se encuentra el pais, y percibir que el sacrilicio o las mayores preslaciones sociales que se concedan ahora representan en realidad un costo menor que la violencia o lalta de cooperación que puede generarse ante la ausencia de tales prestaciones.

Si se pudiera cuantificar fácilmente y demostrar conlundenlemente la relación que exisle entre la falla de seguridad social, laboral, la situación de miseria, elc., y la pérdida en dinero que esle malestar social genera a las empresas, al gobierno y en general a loda la sociedad, probablemenle la concertación sería mucho más lácil de lograr. No se trata de presenlar el logro simulláneo de la eliciencia con equidad como algo cońlictivo. sino como algo necesario, aun cuando en términos realistas y lécnicos el diseño de delerminadas polílicas lendrá que considerar lógicamente los limiles impuestos por eslos dos conceplos.

No se trata tampoco de pensar idealmenle en constituir una sociedad con una distribución perfectamente igualitaria, al contrario a cada quien debe corresponderle de acuerdo a su esfuerzo', pero a todos los que trabajan y sus familias les debe corresponder al menos lo suliciente para vivir con dignidad. Si un trabajador no aporta algo significalivo debido a su poca preparación, actualmente es muy dificil afirmar que sea por causa de su lalta de capacidad e interés, pues en la mayoria de los casos no se ha lenido ni siquiera la opción de decidir si desarrollar o no su potencial.

El ambiente que impera en la comunidad internacional en relación al modelo de desarrollo que debe ser impulsado $y$ al financiamiento condicionante en torno al mismo, lambién es propicio para el tipo de desarrollo que se busca. El condicionamiento ya no estará amparado en torno al conflicto este-oeste, sino que girará en torno a los derechos humanos, a una economía compatible con el mercado, y al equilibrio ecológico. De acuerdo al Partido Social-demócrala (SPD) de Alemania: "debería priorizarse sobre todo la lucha conlra la pobreza absoluta y el explosivo crecimiento poblacional, asi como el fomento de áreas como la

14. Por el carácter de este artículo y por la dificultad de este lema, es comprensible que no se ahonde en como y quién ha de delerminar lo que debe corresponderle a cada individuo. 
educación, la preservación ambiental, los derechos humanos y la democratización' ${ }^{15}$.

\section{Propuesta para un Gestión Macroeconómlca de Consenso}

En este apartado se intentan dar lineamientos generales para llegar a una gestión macroeconómica de consenso, tomando en consideración el lipo de desarrollo al que se aspira, y los coslos sociales generados por el programa de ajuste estructural. Hacemos la salvedad que algunas de las propuestas no son estrictamente de carácter macroeconómico, pero por su relevancia o su relación estrecha con agregados macroeconómicos se ha considerado oportuno sefialarlas.

Antes de entrar a propueslas concretas se planlean a continuación observaciones generales sobre el programa implementado por el gobierno. A este respeclo, podemos decir que las políticas atafien al aumento en la oferta y la productividad han sido seriamente descuidadas, 0 al menos sus resultados han sido escasos; el programa se ha concentrado en las políticas de resiricción a la demanda para solventar desequilibrios macroeconómicos, con todos los costos sociales que implica la disminución del gaslo público. Así, en el combate a la inflación se ha recurrido primordialmenle a la resiricción a la demanda, lo que -junto con factores externos que han permitido una abundancia de divisas y por ende la estabilidad del tipo de cambio nominal $\left.\right|^{10}$ - ha conlribuido a desacelerar la inflación, pero no ha evilado un deterioro de las condiciones de vida de algunos sectores. Pero de cara al fuluro, lo que se necesita es un apara10 productivo moderno, y lasas de crecimiento más allas y sostenibles, que lleven a una disminución gradual pero rápida de la pobreza.

Además, debe aceptarse responsablemente que una economía de mercado no significa que las libres fuerzas del mercado, sin intervención alguna del estado, puedan llevar rápida y sin problema alguno a la eliciencia económica y a la equidad de la sociedad. Si esto fuera el caso. no habria objeto en disculir sobre política económica".

15. Hollz, Uwe, "Piedras de Toque". $D+C$, Desarrollo y Cooperación, Ne $1 /$ 1992.

16. La abundancia de divisas le ha posibilitado al Gobierno apartarse de su meta de mantener el tipo de cambio real, y por consiguiente le ha permitido eliminar las presiones inflacionarias asociadas a la depreciación de la moneda.

17. Este modelo de libre mercado al que algunos aspiran como forma ideal de una economla de tipo capitalista, no existe ni aún en los palses más desarrollados, que al conlrario se caracterizan por una fuerte protección arance- 
Ahora que el clima de paz ha abonado el terreno para introducir politicas económicas que no estén influenciadas por el conflicto bélico, es momenlo propicio de dirigir los esfuerzos a elevar la productividad, y a eliminar las causas más prolundas de la pobreza.

Pasando a propueslas de concertación más concretas, en primer lugar es imprescindible analizar la política fiscal, y negociar eficazmente el peso de las diferentes fuentes de captación de recursos sobre las que debe descansar el sistema fiscal, y la aplicación que se hará del gaslo público. La politica fiscal se toma decisiva por ser uno de los instrumentos más idóneos con que el gobierno cuenta para atacar directarnente la pobreza y redistribuir el ingreso. Al momento de efecluar una reforma fiscal debe regir en todo momento el objelivo de acabar con la pobreza.

Por consiguiente, los ingresos tributarios del estado deben descansar en buena medida en los impuestos que gravan directamente la renta, de manera que los que ganen más contribuyan más a las prestaciones sociales del pals. No se ha de recurrir a impuestos irrealistamente altos pero deben estar vigentes lasa impositivas que, sin constituirse en fuerte freno para la inversión, conserven su carácter progresivo y contribuyan a una buena proporción de los ingresos liscales del eslado ${ }^{16}$. Con la aclual política de reducir las lasas impositivas, el estado está descansando demasiado en la idea de que a menores impueslos, mayor inversión, mayor ingreso y una captación más alla de impuestos ${ }^{10}$. Si esa cadena de sucesos es inlerrumpida en algún punto, junlo con la disminución de los impuestos a la importación - consecuencia de la desgravación arancelaria que simultáneamente ha emiprendido el gobierno, y de la caída de los precios del café - debiliten seriamente la recaudación fiscal.

Para aumentar la contribución fiscal además de analizar las tasas impositivas, es necesario eslablecer un mejor sislema de fiscalización, y

laria y no arancelaria para algunos sectores, y por la existencia de una legislación bastante eficaz para evitar monopolios y otras prácticas desleales en el libre juego de la oferta y la demanda.

18. La negociación de tasas impositivas es un ejemplo claro de como se necesita de la disposición y voluntad de las distintas partes involucradas para llegar efectivamente a una politica tributaria de consenso que pueda realmente ser progresiva. Este tipo de políticas requiere que todas las parles hagan demandas realistas y que no traten de maximizar las ganancias que pueden oblener de esta negociación como sectores.

19. Al respecto no existen estudios contundentes de los resultados que una disminución de tasas impositivas puede lener sobre el ingreso nacional y la captación de impuestos, ni siquiera para el caso de los palses desarrollados. 
lograr la ampliación de la base impositiva de manera que se incluya a aquellas actividades que se delecten que, siendo altamente rentables, no conlribuyan lo suficiente a la recaudación tributaria. Simultáneamente, debe realizarse una oportuna inversión pública, medida que varios estudios indican contribuye significativamente al tomento de la inversión privada.

En relación a la aplicación del gasto público, es preciso asegurar que no se pueda disminuir la proporción que representa el gaslo en programas sociales dentro del gasto público total de forma unilateral. La inversión en el recurso humano, sobre todo a través de la educación y capacitación electiva, debe verse como una polílica de oferta deslinada a aumentar la produclividad a mediano plazo. Sólo con una mano de obra especializada, y que tenga satisfecha sus necesidades básicas, podrá aumentarse cualitativa y cuanlilativamente la producción en forma sostenida.

Los programas sociales contra la pobreza deben ser prolegidos aún a cosla de otras allernativas. Ahora es momento propicio para negociar que la mayor parte de fondos que anles se dedicaba a gastos militares se dediquen a gaslos sociales. Para plantear demandas concrelas hay que considerar el monto lotal de los fondos necesarios para impulsar programas sociales baslante efectivos, asi como también la proporción máxima que llegó a alcanzar el gasto social dentro del gasto total antes del conflicto armado, el monto de otros proyectos prioritarios, y los recursos con que se cuenta.

En cuanto a los organismos que lendrán a su cargo el desarrollo de programas con senlido social, las ONG podrian demandar una participación activa en este campo, aún cuando supervisados por el gobiemo. Si no exisle una luerte politización en las actividades de las ONG, el gobierno saldria favorecido al aprovechar la experiencia de estas organizaciones para delectar las necesidades más urgentes de las comunidades, y la forma de como resolverlas.

Una política de gasto público con sentido humano puede reducir los cosios sociales asociados a las polílicas de ajuste esiructural. En este sentido, lo que se pretende es que la reducción de déficit fiscal se logre a través de un aumento en la recaudación tributaria, de la disminución de los gaslos en delensa y de otros gastos improductivos, pero con un aumento de los fondos destinados a los servicios sociales.

Puede solicitarse asimismo la creación de una dependencia encargada de auditar la caplación y uso de fondos estatales. Esle organismo deberá estar desvinculado de todo nexo político, y aún cuando no tuviera 
poder decisorio sobre el uso que debe hacerse de los fondos, si debe contar con la suficiemle auloridad para fiscalizar y al mismo liempo evitar el despilfarro de recursos, y la existencia de gobiernos corruptos e ineficientes. Los inlormes de auditoria deberian ser publicados para que toda persona interesada tuviera acceso a ellos, y en general toda información proveniente del sector público no deberla ser privilegio de unos pocos.

En cuanlo a la política de desgravación arancelaria, debe adoptarse el compromiso que aún en el caso de zonas libres o uniones aduaneras, ciertos bienes considerados de lujo no eslén exentos de impuestos. igualmente, si el resto de paises olorgaran incenlivos a sus productos de exporlacióno aplicaran barreras arancelarias o no arancelarias a nuestros productos, podría eslar abierla la posibilidad de recurrir al mismo trato para los bienes provenientes de esas naciones, cuando la defensa de fuentes de trabajo en el país asi lo requiera.

Es necesario que además de una política crediticia concesional para el sector reformado, el seclor informal y las actividades produclivas en las zonas que fueron tradicionalmenle conflictivas, existe otro tipo de asistencia sobre lodo técnica, de capacilación, y de mercadeo y comercialización para ayudar al despegue de eslas aclividades y para que puedan insertarse exitosamente no sólo al mercado interno sino lambién al mercado internacional, 10 que puede significar recurrir en su inicio a subvenciones temporales.

Para preservar los salarios reales de los trabajadores debe negociarse que el salario mínimo establecido tome en cuenta no sólo la productividad (concepto ditícil de medir) sino también, y sobretodo, que considere el costo aclualizado de la canasla ampliada. La revisión salarial debería realizarse periódicamente, aunque en diferentes momentos para los distinios sectores, para evitar presiones inllacionarias generalizadas. Estamos conscienles de que la recuperación de los salarios reales sólo podrá hacerse en forma gradual, y que por otro lado la indezación de los salarios podría originar una espiral inflacionaria dilícil de controlar, que rápidamente eliminaria cualquier mejora salarial, pero todo empresario en el momenlo de fijar el salario a sus empleados debe tomar en cuenla no sólo su lasa de ganancia ${ }^{20}$ sino lambién la condición de vida a que está destinando a ese trabajador y su familia.

20. La tasa de ganancia es un dato muy importante por cuanto permite calcular hasta cierto punto la posibilidad real de incrementos salariales sin menoscabo de la actividad productiva, y también posibilita comparar esla tasa co la tasa promedio de los palses industrializados, pero el conocimiento de este dalo también puede generar un fuerte manipuleo político y de clases. 
En cuanto a la politica de control de precios, es conveniente concertar la existencia de leyes antimonopólicas efectivas y la creación de un organismo de defensa al consumidor que tuviera dentro de sus atribuciones la publicación periódica de la lista de precios de productos básicos de similar calidad ofrecidos en distintos establecimientos, y la denuncia responsable de actividades oligopólicas o monopólicas.

El Eslado puede además implementar medidas incentivadoras (cierta exención de impueslos por ejemplo) para aquellas empresas que ofrecieran productos básicos a precio de costo o subsidiados a sus trabajadores a través de despensas, o que ofrecieran participación de utilidades a sus trabajadores.

Por los electos inflacionarios que surgen de toda devaluación, puede concerlarse que lal medida vaya acompanada de otras acciones que contribuyan a manlener el poder adquisitivo de la clase trabajadora. Aqui puede pensarse en algún lipo de subsidio dirigido a grupos especificos para ciertos productos que forman parte de la canasta de consumo de los más pobres. La electividad de tal medida dependerá de que tan bien pueda hacer llegar esle subsidio a la clase que se pretende favorecer.

En lorno al aumento de las tarifas públicas, puede plantearse la alternaliva de que el aumento se haga en forma gradual, y que además se mantengan larifas diterenciadas para los servicios de agua y luz de acuerdo a la cantidad consumida (para evitar despilfarros), a la zona geográfica (zonas residenciales, barrios pobres, etc.), y según el uso (privado, industrial, comercial, etc.).

Otras medidas, no precisamente de política macroeconómica que aunque no resuelven la pobreza pueden contribuir a aliviarla a corlo plazo, y que por tanto hay que proponer, influyen una campana más agresiva de control de la nalalidad, y la creación de guarderias ${ }^{21}$, la alimentación de nifios en las escuelas, mejores pensiones, un sistema eficiente de protección a la vejez, elc.

De lo anterior podemos concluir que si bien se pueden negociar determinadas políticas económicas, para solvenlar la pobreza se requiere además de una serie de medidas que incluyen sobretodo mejores preslaciones sociales, mejoras en la capacilación humana, mejor distribución del ingreso, mejor distribución y mayor acceso a la riqueza, parte de lodo un paquete de políticas secioriales, de polílicas sociales y de reformas institucionales, administrativas y legales. Pero sobretodo se necesita de

21. Evidencia de que la falta de este tipo de políticas contribuye a agravar la pobreza se encuentra en Briones, Carlos, Op. Cit. 
los mecanismos adecuados para lograr una correlación de fuerzas equilibrada y del deseo de los distimlos antes participalivos para lograr que todo lo que formalmente se acepta o lo que dentro del marco legal se acuerde, se lleve a la práctica electivamente, y que no se obvien o se distorsionen las decisiones o politicas que se adopten, causa frecuenle en el pasado de que los deseos expresados por los distintos gobiemos hayan lenido nulos o escasos resultados en la lucha contra la pobreza. 